\title{
The importance of the five phosphoribosyl- pyrophosphate synthetase (Prs) gene products of Saccharomyces cerevisiae in the maintenance of cell integrity and the subcellular localization of Prs1p
}

\author{
Roger Schneiter, ${ }^{2}$ Andrew T. Carter, ${ }^{3}$ Yolanda Hernando, ${ }^{3}$ \\ Günther Zellnig, ${ }^{4}$ Lilian M. Schweizer ${ }^{1}$ and Michael Schweizer ${ }^{1}$
}

Author for correspondence: Michael Schweizer. Tel: +44 131451 3186. Fax: +44 1314513009. e-mail: M.Schweizer@hw.ac.uk

1 Department of Biological Sciences, Heriot-Watt University, Edinburgh EH14 4AS, UK

2 Institut für Biochemie und Lebensmittelchemie, Technische Universität Graz, Petersgasse 12/II, A-8010 Graz, Austria

3 Genetics and Microbiology Dept, Institute of Food Research, Norwich Research Park, Colney, Norwich NR4 7UA, UK

4 Institut für Pflanzenphysiologie, KarlFranzens Universität, Schubertstrasse 51, A-8010 Graz, Austria
Phosphoribosyl-pyrophosphate synthetase (Prs) catalyses the synthesis of phosphoribosyl pyrophosphate (PRPP), an intermediate in nucleotide metabolism and the biosynthesis of the amino acids histidine and tryptophan. The Saccharomyces cerevisiae genome contains a family of five PRS genes, PRS1-PRS5. Using anti-peptide antisera directed against two different epitopes of Prs1p it was shown that Prs1p localizes to granular cytoplasmic structures. This localization was confirmed by living cell microscopy of strains expressing a functional green fluorescent protein (GFP)-tagged Prs1p. Analysis of Prs1p distribution in conditional secretory-deficient (sec) mutants suggested that the observed distribution of Prs1p is independent of the secretory pathway. Electron microscopy revealed that plasma membrane invaginations and accumulation of cytoplasmic vesicles were more frequent in strains which lack some of the PRS genes than in the wild-type. The fact that $\Delta$ prs 1 and $\Delta$ prs 3 are hypersensitive to caffeine and unable to recover from exposure to it as judged by the release of alkaline phosphatase points to a possible link between Prs and the maintenance of cell integrity.

Keywords: phosphoribosyl-pyrophosphate synthetase (Prs), subcellular localization of Prs1p, cell integrity, Saccharomyces cerevisiae

\section{INTRODUCTION}

Phosphoribosyl-pyrophosphate synthetase (Prs; ATP: D-ribose-5-phosphate pyrophosphotransferase; EC 2.7.6.1) catalyses the transfer of the terminal pyrophosphoryl group of ATP to ribose 5-phosphate to generate 5-phosphoribosyl 1( $\alpha$ )-pyrophosphate (PRPP). PRPP is required for the synthesis of purine and pyrimidine nucleotides, the pyridine nucleotide cofactor $\mathrm{NAD}(\mathrm{P})$ and the amino acids histidine and tryptophan. In nucleotide synthesis, PRPP is used both for de novo synthesis and for the salvage pathway, by which bases

\footnotetext{
Abbreviations: DIC, differential interference contrast; 5-FOA, 5-fluoroorotic acid; GFP, green fluorescent protein; NHR, non-homologous region; PRPP, 5-phosphoribosyl 1( $\alpha$ )-pyrophosphate; Prs, 5-phosphoribosyl-1( $\alpha$ pyrophosphate synthetase; RLU, relative light units.
}

are metabolized to nucleotides (Hove-Jensen, 1989; Khorana et al., 1958; Switzer, 1969; Switzer \& Sogin, 1973; Tatibana, 1995). Prs is thus a central enzyme in the metabolism of nitrogen-containing compounds.

Cells are impermeable to PRPP. However, Eschericha coli prs mutants are fully rescued if supplemented with purine, pyrimidine, pyridine nucleotides, histidine and tryptophan (Hove-Jensen, 1989), indicating that in this organism Prs is not required for a function other than PRPP synthesis, although it has been suggested that the enzyme may be implicated in the initiation of DNA replication in E. coli (Sakakibara, 1992). In humans, superactivity of Prs correlates with gouty arthritis and in some cases, disorders of the nervous system (Becker et al., 1988, 1995).

PRS genes have been cloned and sequenced from 19 organisms; both rat and human have two ubiquitously 
Table 1. Yeast strains used in this study

\begin{tabular}{|c|c|c|}
\hline Strain & Genotype & Reference* \\
\hline $\mathrm{X}-2180-\mathrm{IA}$ & MATa CUP1 gal2 mal SUC2 & G. Daum \\
\hline Tetraploid & $M A T \mathbf{a} / M A T \mathbf{a} M A T \alpha / M A T \alpha$ & Kadowaki et al. (1995) \\
\hline YPH501 & $\begin{array}{l}\text { MATa/ } \alpha \text { ade2-101 ura3-52 bis3-200 leu2-1 } \\
\text { lys2-801 trp1-63 }\end{array}$ & Sikorski \& Hieter (1989) \\
\hline $\sec 7-1$ & $M A T ? \sec 7-1$ & G. Daum \\
\hline $\sec 15-1$ & MAT? $\sec 15-1$ & G. Daum \\
\hline $\sec 16-2$ & MAT? $\sec 16-1$ & G. Daum \\
\hline YN94-1 & $\begin{array}{l}\text { MAT a ade2-1 his3-11 leu2-3,112 trp1-1 ura3-1 } \\
\text { can1-100 ssd1-d2 GAL }\end{array}$ & Hernando et al. (1999) \\
\hline YN94-2 & $\begin{array}{l}\text { MATa ade2-1 his3-11 leu2-3,112 trp1-1 ura3-1 } \\
\text { can1-100 ssd1-d2 GAL }\end{array}$ & Hernando et al. (1999) \\
\hline YN94-4 & YN94-1 $\Delta p r s 1:: H I S 3$ & Carter et al. (1997) \\
\hline YN94-200 & YN94-1 $\Delta$ prs1::HIS3(pYES-PRS1) & Carter et al. (1997) \\
\hline YN94-18 & YN94-1 $\Delta p r s 3:: T R P 1$ & Carter et al. (1997) \\
\hline YN94-19 & YN94-1 $\Delta p r s 2:: U R A 3$ & Carter et al. (1997) \\
\hline YN94-20 & YN94-1 $\Delta p r s 4:: L E U 2$ & Carter et al. (1997) \\
\hline YN96-1 & YN94-1 $\Delta$ prs $5:$ :KanMX4 & Laboratory collection \\
\hline YN94-12 & YN94-1 $\Delta p r s 1:: H I S 3 \Delta p r s 3::$ TRP1 & Laboratory collection \\
\hline YN94-14 & YN94-1 $\Delta p r s 2:: U R A 3 \Delta p r s 3::$ TRP1 & Laboratory collection \\
\hline YN94-22 & YN94-1 $\Delta p r s 1:: H I S 3 \Delta p r s 4:: L E U 2$ & Laboratory collection \\
\hline YN95-21 & YN94-1 $\Delta p r s 1:: U R A 3$ & Laboratory collection \\
\hline YN96-3 & YN94-1 $\Delta p r s 2:: U R A 3 \Delta p r s 5:: k a n M X 4$ & Laboratory collection \\
\hline YN96-5 & YN94-1 $\Delta$ prs4::LEU2 $\Delta p r s 5::$ kanMX4 & Laboratory collection \\
\hline YN96-52 & YN94-1 $\Delta$ prs3::kanMX4-IoxP & Laboratory collection \\
\hline YN97-141 & YN94-1 $\Delta$ prs2::kanMX4-IoxP & Laboratory collection \\
\hline YN95-29 & YN94-1 $\Delta p r s 1:: H I S 3 \Delta p r s 2:: U R A 3$ & Laboratory collection \\
\hline YN95-100 & YN94-1 $\Delta p r s 3::$ TRP1 $\Delta p r s 4:: L E U 2$ & Hernando et al. (1999) \\
\hline YN95-123 & YN94-2 $\Delta p r s 2:: U R A 3 \Delta p r s 4:: L E U 2$ & Laboratory collection \\
\hline YN95-25 & YN94-1 $\Delta p r s 1:: H I S 3 \Delta p r s 3::$ TRP1 $\Delta p r s 4::$ LEU2 & Laboratory collection \\
\hline YN95-26 & YN94-1 $\Delta p r s 1:: H I S 3 \Delta p r s 2:: U R A 3 \Delta p r s 3::$ TRP1 & Laboratory collection \\
\hline YN96-72 & $\begin{array}{l}\text { YN94-1 } \Delta p r s 2:: U R A 3 \Delta p r s 4:: L E U 2 \\
\Delta p r s 5:: \text { kanMX4 }\end{array}$ & Laboratory collection \\
\hline YN97-96 & YN94-1 $\triangle p r s 1::$ GFP-PRS1 & This study \\
\hline YN98-8 & YN94-1 $\Delta p r s 1:$ : GFP-PRS1 $\Delta p r s 2::$ kanMX4-IoxP & This study \\
\hline YN98-10 & YN94-1 $\Delta p r s 1:$ :GFP-PRS1 $\Delta p r s 3::$ kanMX4-IoxP & This study \\
\hline YN98-12 & YN94-1 $\Delta p r s 1:$ :GFP-PRS1 $\Delta p r s 4::$ kanMX4-IoxP & This study \\
\hline YN98-14 & YN94-1 $\Delta p r s 1::$ GFP-PRS1 $\Delta p r s 5::$ kanMX4-IoxP & This study \\
\hline
\end{tabular}

*G. Daum, Technical University of Graz, Austria.

expressed PRS genes, with a third testis-specific gene found in man (Taira et al., 1990). Saccharomyces cerevisiae harbours five unlinked structural genes encoding Prs polypeptides Prs1p-Prs5p. Within this PRS multigene family, PRS2, PRS3 and PRS4 exhibit more sequence similarity to each other than to either PRS1 or PRS5 (Carter et al., 1994, 1997; Hernando et al., 1998, 1999). Characterization of yeast strains deleted individually for each of the PRS genes indicates that, although none of them is essential for viability, the contribution of each of the gene products to the metabolic status of the cell appears to differ both qualitatively and quantitatively. Disruption of PRS1 or PRS3 results in a severely reduced growth rate and markedly decreased total Prs enzyme activity (Carter et al., 1997; Hernando et al., 1999), suggesting that these polypeptides have a key structural or regulatory role in PRPP synthesis.

The predicted Prs polypeptides Prs $2 p$, Prs $3 p$ and Prs4p are 318-355 amino acids long and have molecular masses of $34 \cdot 7,35 \cdot 1$ and $39 \cdot 0 \mathrm{kDa}$, respectively. Prs1p (427 amino acids, $47 \cdot 0 \mathrm{kDa}$ ) contains a unique in-frame insertion of 105 amino acids, termed the non-homologous region (NHR) 1-1. Prs5p possesses two NHRs, one of 116 amino acids (NHR5-2) located between the divalent-cation-binding site and the PRPPbinding site, as is the case for NHR1-1 present in Prs1p. The other NHR, NHR5-1, consisting of 70 amino acids, is located directly in front of the divalent-cation- 
binding site and thus Prs5p with 496 amino acids is the largest polypeptide of the family (predicted molecular mass $53.5 \mathrm{kDa}$ ). The NHRs are not similar to each other or to any other sequences in the databases. The significance and function of the NHRs is at present unknown.

In the present work, by using antisera against two different epitopes of Prs1p, one outside (AK41) and the other within (AK39) the NHR1-1 of Prs1p, we determined the subcellular distribution of Prs1p by immunofluorescence analysis and by cellular fractionation. In fixed cells, both antisera associated with cytoplasmic structures. In living cells, a functional green fluorescent protein (GFP)-Prs1p fusion also localized to granular cytoplasmic structures. The fact that certain PRS deletants are hypersensitive to caffeine and respond to the addition of caffeine to a liquid culture by releasing alkaline phosphatase more readily into the medium than the wild-type points to a possible relationship between Prs and the structure and/or function of the cell wall.

\section{METHODS}

Yeast strains and culture conditions. The yeast strains used in this study are listed in Table 1 . Strains were cultured at the temperature indicated in either YEPD (1\% yeast extract, $2 \%$ Bacto peptone, $2 \%$ glucose) or synthetic minimal medium supplemented with appropriate amino acids and glucose, galactose or lactate (Kaiser et al., 1994). Caffeine plates were prepared by combining equal volumes of double-strength YEPD agar and a double-strength aqueous solution of the final caffeine concentration required. 5-Fluoroorotic acid (5-FOA; Sigma) plates were prepared as described by Boeke et al. (1987).

Construction of GFP-tagged Prs1p yeast strains. The $0.7 \mathrm{~kb}$ S65T gene (Heim \& Tsien, 1996) was amplified by PCR from pFA6a-KanMX6 as template (Wach et al., 1997) using the tailed primers $\mathrm{P} 1,5^{\prime}{ }^{-36(\mathrm{PRS} 1)}\left[5^{\prime}\right.$-TACCGTTTAATAGTCTTAATTTGAAGAGAAATAAAAATG ${ }^{+3(\mathrm{PRS} 1)+4(\mathrm{GFP})}$ AGTAAAGGAGAAGAACTTTTC-3' $]^{+24(\mathrm{GFP})}$, and GFP-end, +18(PRS1)$\left[5^{\prime}\right.$-AATTTTACATTACG ${ }^{+4(\mathrm{PRS} 1)+714(\mathrm{GFP})}$ TTTGTATAGTTCATCCATGCC-3 $\left.3^{\prime}\right]^{+694(\mathrm{GFP})}$. Positions of the primers in PRS1 and GFP are indicated by superscripts and the 765 bp product includes nt -36 to +3 of the PRS1 nucleotide sequence (Carter et al., 1994) fused to the second codon of GFP and $15 \mathrm{nt}$ of sequence complementary to codons 2-6 of PRS1 fused to the $3^{\prime}$ end of GFP lacking the stop codon.

A 909 bp fragment of PRS1 was amplified by PCR using YN94-1 DNA as template with the primers PRS1 start, ${ }^{+4}$ (PRs1) $\left[5^{\prime}\right.$-CGTAAGTGTAAAATTTTTGT-3'] ${ }^{+23(\text { Prs1) }}$, and

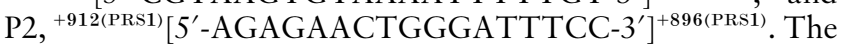
$5^{\prime}$ end of the amplification product starts at the second codon of PRS1 and was therefore complementary to the $3^{\prime}$ end of the GFP PCR product.

Both PCR products were joined in a PCR-ligation reaction (Pearson et al., 1998) driven with an excess of the two outer primers, P1 and P2. To optimize the efficiency of integration of the $1659 \mathrm{bp}$ GFP-PRS1' construct the length of the $5^{\prime}$ flanking region was increased. A $190 \mathrm{bp}$ fragment of PRS1 $5^{\prime}$-flanking sequence was amplified using primers P11,

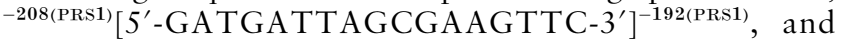

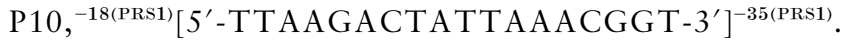
By virtue of the 18 bp overlap with the $5^{\prime}$ end of the GFP-PRS1' construct, the two products were fused by PCR using the primers P11 and P2 to give rise to the final product of $1831 \mathrm{bp}$. This PCR product was used after agarose gel electrophoresis purification (Sambrook et al., 1989) to transform YN95-21 (Gietz \& Woods, 1994) successfully to 5-FOA resistance, creating the strain YN97-96.

YN97-96 carrying $\triangle p r s 1:: G F P-P R S 1$ was used to create four additional strains, individually deleted for PRS2, PRS3, PRS4 and PRS5. YN98-8 and YN98-10, which are deleted for PRS2 and PRS3, respectively, were created by transforming YN9796 with the PCR products obtained from YN97-141 and YN96-52 using primers flanking the disrupted $P R S 2$ and $P R S 3$ loci (Carter et al., 1997). YN98-12 and YN98-14 were created by the short flanking homology method (Wach et al., 1994) using appropriate primers for the flanking regions of $P R S 4$ and PRS5 in conjunction with pUG6 DNA (Güldener et al., 1996) as the template. Deletions were checked by PCR and Southern blot analysis.

Microscopic techniques and immunofluoresecence. For immunofluorescence, cells in the early exponential phase of growth were fixed in formaldehyde/methanol exactly as described by Wente et al. (1992). Antisera AK39 (peptide 1) and AK41 (peptide 2), described by Carter et al. (1997), were pre-adsorbed onto fixed cells and subsequently used at a 1:2700 (AK39) and a 1:900 (AK41) dilution. Binding of AK39 or AK41 was detected using FITC-conjugated secondary antibody against rabbit IgG (Oncogene Science Diagnostics; diluted 1:100). Invertase was detected using a 1:2500 dilution of a rabbit anti-invertase antiserum generously provided by R. Schekman, Berkely, CA. Nucleic acid was stained by DAPI (4',6-diamidino-2-phenylindole dihydrochloride). Epifluorescence analysis of wild-type and haploid strains deleted for the PRS genes indicated was performed using a Zeiss Axiovert 35 microscope (Carl Zeiss) and photographs were taken using a $\times 100$ objective with Kodak T-Max 100 PRO (Eastman Kodak).

Laser scanning microscopic analysis of tetraploid and haploid cells was performed using a Leica TCS $4 \mathrm{~d}$ microscope and photographs were taken with a PL APO $63 \times 1 / 1.40$ objective on Kodak Ektachrome Elite 100 slide film (Eastman Kodak). Corresponding pictures were recorded with identical pinhole openings and amplification settings and were printed with the same exposure settings.

For electron microscopical investigations, cells were fixed in $4 \%$ paraformaldehyde $/ 5 \%$ glutaraldehyde in $0 \cdot 1 \mathrm{M}$ cacodylate buffer $\mathrm{pH} 7 \cdot 0$ and $1 \mathrm{mM} \mathrm{CaCl}$ for $90 \mathrm{~min}$ at room temperature. Cells were then washed in buffer with $1 \mathrm{mM}$ $\mathrm{CaCl}_{2}$ for $1 \mathrm{~h}$ and incubated for $1 \mathrm{~h}$ with a $2 \%$ aqueous solution of $\mathrm{KMnO}_{4}$. Fixed cells were washed in distilled $\mathrm{H}_{2} \mathrm{O}$ for $30 \mathrm{~min}$ and incubated in $1 \%$ sodium metaperiodate for $20 \mathrm{~min}$. Samples were then rinsed in distilled $\mathrm{H}_{2} \mathrm{O}$ for $15 \mathrm{~min}$ and post-fixed for $2 \mathrm{~h}$ in $2 \% \mathrm{OsO}_{4}$ buffered with $0 \cdot 1 \mathrm{M}$ cacodylate at $\mathrm{pH} 7 \cdot 0$. After another wash with buffer for $30 \mathrm{~min}$, the samples were dehydrated in a graded series of ethanol washes $(50-100 \%$ with en bloc staining in $2 \%$ uranylactetate in $70 \%$ ethanol overnight) and embedded in Spurr resin. Ultrathin sections were stained with lead citrate and viewed with a Philips CM 10 electron microscope.

Subcellular fractionation. Wild-type strain X2180-1A was used for fractionation experiments. One litre cultures in 2 litre flasks were incubated at $30^{\circ} \mathrm{C}$ on a rotary shaker with vigorous aeration. For isolation of the plasma membrane, 
Golgi, nuclei and cytosol, cells were grown in YEPD. Mitochondria and microsomes were isolated from cells grown in $2 \%$ lactate medium. Cells were harvested by centrifugation and converted to spheroplasts as described by Daum et al. (1982).

Subcellular fractionation was performed essentially as reviewed by Zinser \& Daum (1995). Briefly, plasma membranes were isolated according to Serrano (1988) and approximately 100 -fold enriched for plasma membrane $\mathrm{H}^{+}$-ATPase. Mitochondria were isolated according to Daum et al. (1982) and were approximately 4-fold enriched for the major protein of the outer mitochondrial membrane, porin. Microsomes were obtained after clearing the post-mitochondrial supernatant as described by Gaigg et al. (1995). Isolation of the Kex2penriched (200-fold) Golgi fraction was as described by Leber $e t$ al. (1995). Nuclei were isolated by sucrose density-gradient centrifugation as described by Hurt et al. (1988) and approximately 12-fold enriched for the ER marker BiP/Kar2p. Cytosol was obtained by $40 \%\left(\mathrm{NH}_{4}\right)_{2} \mathrm{SO}_{4}$ precipitation of the $10000 \mathrm{~g}$ supernatant obtained after removal of the crude nuclear pellet.

Protein and Western blot analysis. Prior to quantification by the Lowry method, proteins were precipitated with $10 \%$ trichloroacetic acid and solubilized in $0 \cdot 1 \%$ SDS, $0 \cdot 1 \mathrm{M}$ $\mathrm{NaOH}$. Proteins were separated on a $10 \%$ SDS-PAGE gel and transferred to nitrocellulose filters (Hybond-C; Amersham Pharmacia Biotech). After blocking, blots were incubated at $4{ }^{\circ} \mathrm{C}$ overnight in a 1:2000 dilution of AK39 or AK41. The intensity of the immunoreaction was quantified after scanning the autoradiogram by using the 'wand tool' present in the image analysis software NIH Image 1.60. Antisera against yeast plasma membrane $\mathrm{H}^{+}$-ATPase, Kex2 protease and $\mathrm{BiP} /$ Kar2p were obtained from G. Daum (Technical University of Graz, Austria) and were originally gifts from R. Serrano (Universidad Politecnica, Valencia, Spain), R. Fuller (University of Michigan Medical Center, Ann Arbor, MI, USA) and R. Schekman (University of California, Berkeley, CA, USA), respectively. Antisera against porin and the $40 \mathrm{kDa}$ microsomal protein were obtained from G. Daum.

Caffeine sensitivity. A single colony from a freshly grown YEPD culture was used to inoculate $10 \mathrm{ml}$ YEPD broth. Cultures were grown overnight in a vertical rotary incubator. A constant inoculum of $2 \times 10^{4}$ cells was applied by means of a multiple inoculator on YEPD plates containing caffeine at the concentrations indicated. Plates were scored after incubation at $30^{\circ} \mathrm{C}$ for $3 \mathrm{~d}$.

Chemiluminescent determination of alkaline phosphatase release. Overnight $10 \mathrm{ml}$ cultures obtained as above were used to inoculate $200 \mathrm{ml}$ YEPD broth in $500 \mathrm{ml}$ Erlenmeyer flasks. Cultures were then shaken in an orbital incubator until an $\mathrm{OD}_{600}$ of 1.0 was reached, at which time either $10 \mathrm{ml}$ of a $100 \mathrm{mM}$ caffeine stock solution or $10 \mathrm{ml}$ sterile distilled $\mathrm{H}_{2} \mathrm{O}$ was added. One millilitre samples were collected immediately and then at hourly intervals and the cells removed by centrifugation (13000 r.p.m., 3 min). Supernatants were stored at $-20{ }^{\circ} \mathrm{C}$ until the assay was carried out. Alkaline phosphatase was assayed using a chemiluminescent detection reagent (Lumiphos-530 AP detection reagent; Amersham Pharmacia Biotech). One hundred microlitres of supernatant was mixed with an equal volume of Lumiphos-530 in a $5 \mathrm{ml}$ plastic luminometer tube (Sarstedt) and incubated at room temperature. Relative light units (RLU) were measured using a Lumat LB 9501 luminometer (Berthold). Maximum RLU readings were obtained after $45-60 \mathrm{~min}$, therefore reaction rate $\left(\mathrm{RLU} \mathrm{min} \mathrm{m}^{-1}\right)$ was calculated from the linear part of a plot of several readings recorded between 10 and 90 min after the addition of the reagent.

\section{RESULTS}

\section{Determination of the intracellular distribution of Prs1p by confocal laser scanning immunofluorescence}

The specificity of the antisera AK39 raised against a peptide that is part of the NHR1-1 of Prs1p (residues 272-288) and AK41 raised against the peptide sequence of Prs1p from residues 120 to 136 was tested with protein extracts from cells that express Prs1p under the transcriptional control of a galactose-inducible GAL1 promoter in YN94-200 [Aprs1::HIS3(pYES-PRS1)] (Carter et al., 1997). Both antisera recognize on SDSPAGE gels a single band with a molecular mass of $54 \mathrm{kDa}$ which is only visible in galactose-containing medium, suggesting that both antisera recognize only Prs1p. The specificity of the antisera was also confirmed by immunofluorescence using fixed cells of YN94-200, when a signal was obtained only when the strain was grown in galactose (data not shown).

Preliminary analysis of the subcellular distribution of Prs1p in cells which overexpress it suggested that it is located in the cytoplasm. Overexpression of a protein can, however, potentially affect its subcellular distribution. We therefore analysed the distribution of Prs1p in wild-type cells. Since high-resolution laser scanning immunofluorescence analysis is limited by the small size of haploid yeast cells, large tetraploid cells were employed to allow detection of Prs1p at its normal level of expression (Fig. 1). This analysis revealed that both AK39 and AK41 antisera stain the cytoplasm in a granular pattern. This distribution is not specific for the tetraploid strain used since the same granular staining was also observed if diploid wild-type cells (YPH501; Table 1) were stained with the antisera.

\section{Prs1p distribution is not altered in secretory mutants}

To determine whether the granular structures referred to above represent a post-Golgi compartment, Prs1p distribution was analysed in secretory (sec) mutants. For these experiments we chose sec16, sec7 and sec15 temperature-sensitive mutants that are blocked in protein transport from the ER, protein transport through the Golgi and consumption of Golgi-derived vesicles at the plasma membrane, respectively (Novick et al., 1980; Rapoport et al., 1996; Schekman, 1985). These strains were cultured in YEPD medium at $23{ }^{\circ} \mathrm{C}$ and shifted to $37^{\circ} \mathrm{C}$ and into low glucose $(0 \cdot 1 \%)$ YEPD medium so as to simultaneously induce de novo synthesis of the marker protein secretory glycoprotein invertase. Two hours after the shift, the cells were examined by immunofluorescence using antisera against invertase and AK39. The subcellular distribution of Prs and invertase in the sec7-1 mutant cells is shown in Fig. 2. The invertase staining pattern observed for the 

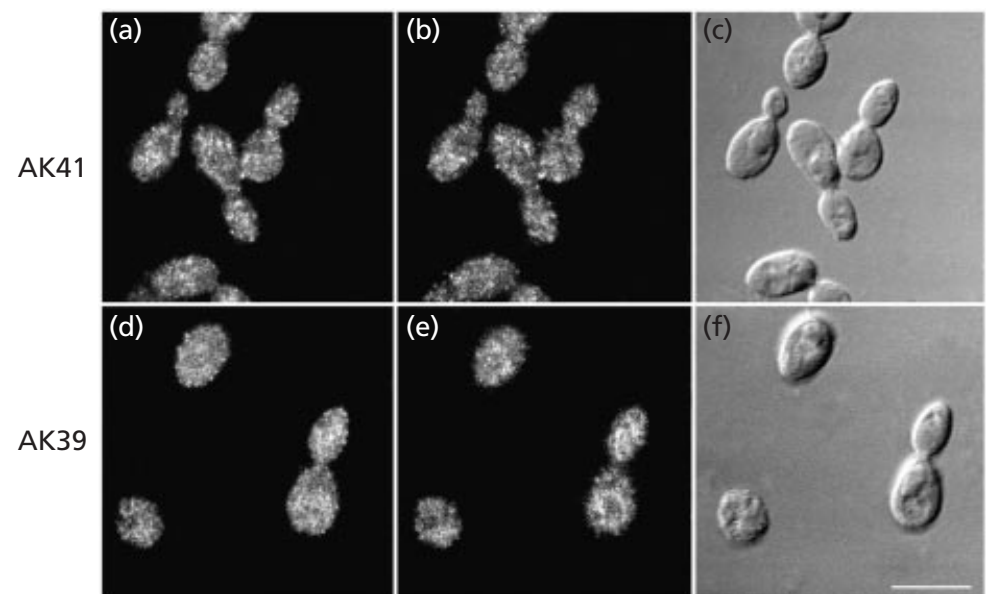

Fig. 1. Confocal analysis of Prs1p distribution in tetraploid wild-type cells. Cells were grown to early exponential phase in YEPD, fixed and stained with AK41 (a-c) or AK39 (d-f). Two serial $0.5 \mathrm{~mm}$ thick confocal sections are shown in (a) and (b) and in (d) and (e). DIC pictures of the same visual fields are shown in (c) and (f). Bar, $5 \mu \mathrm{m}$.
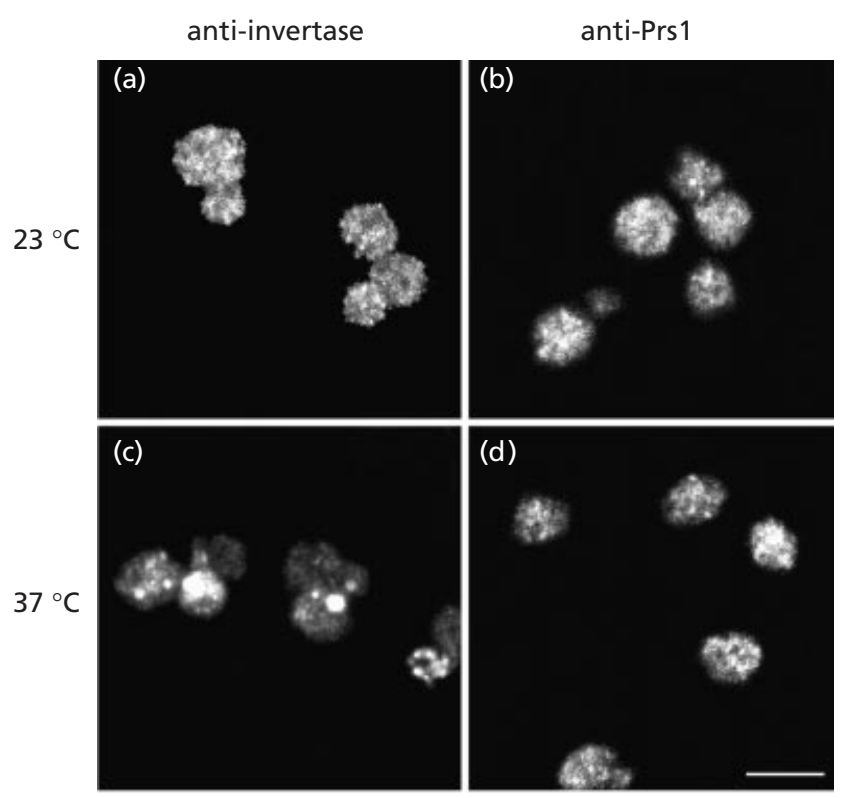

Fig. 2. Prsip localization is independent of the secretory pathway. sec7-1 temperature-sensitive haploid cells were grown to early exponential phase in YEPD. Cultures were split, shifted to low glucose medium ( $0.1 \%$ glucose in YEPD) and incubated at 23 or $37^{\circ} \mathrm{C}$ for $2 \mathrm{~h}$. Cells were fixed and stained with antisera against invertase $(a, c)$ or anti-Prs1p (AK39; b, d). Cells incubated at the permissive temperature $\left(23^{\circ} \mathrm{C}\right)$ are shown in (a) and (b); cells shifted to $37^{\circ} \mathrm{C}$ are shown in (c) and (d). Bar, $5 \mu \mathrm{m}$.

sec7-1 cells at the permissive temperature (Fig. 2a) changed after shifting to the restrictive temperature to the typical punctate one characteristic of invertase accumulation in the Golgi (Fig. 2c) (Whitters et al., 1994). The granular anti-Prs1p staining of sec7-1 mutants grown at restrictive conditions, on the other hand, was not different from that of cells incubated at the permissive temperature (Fig. 2b, d) suggesting that Prs1p

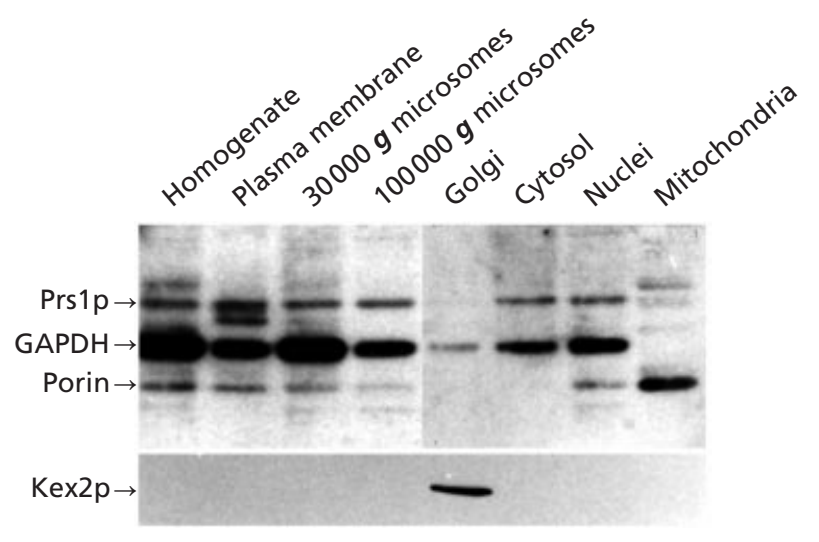

Fig. 3. Subcellular distribution of Prs1p. Ten microgrammes of protein of the indicated fractions was separated by $10 \%$ SDSPAGE, transferred to a nitrocellulose membrane and probed sequentially with antisera AK39 against Prs1p (54 kDa), glyceraldehyde-3-phosphate dehydrogenase (GAPDH, $43 \mathrm{kDa})$, porin $(30 \mathrm{kDa})$ and $\mathrm{Kex} 2 \mathrm{p}(130 \mathrm{kDa}$; signal shown from a different exposure of the same blot). The lane representing the Golgi fraction contains only $1 \mu \mathrm{g}$ protein.

localization is independent of the secretory pathway and that the granular Prs staining does not represent a postGolgi compartment. Consistent with this conclusion no alteration of Prs1p distribution was observed in sec16 and $\sec 15$ mutants (data not shown).

\section{Distribution of Prs1p in subcellular fractions}

The possible association of Prs1p with any membranebordered subcellular compartment was analysed by subcellular fractionation. To this end, yeast subcellular fractions enriched for marker proteins of the plasma membrane (plasma membrane $\mathrm{H}^{+}$-ATPase, 100-fold enriched), $30000 \mathrm{~g}$ microsomes (2-fold enriched for a $40 \mathrm{kDa}$ microsomal protein), $100000 \mathrm{~g}$ microsomes, 

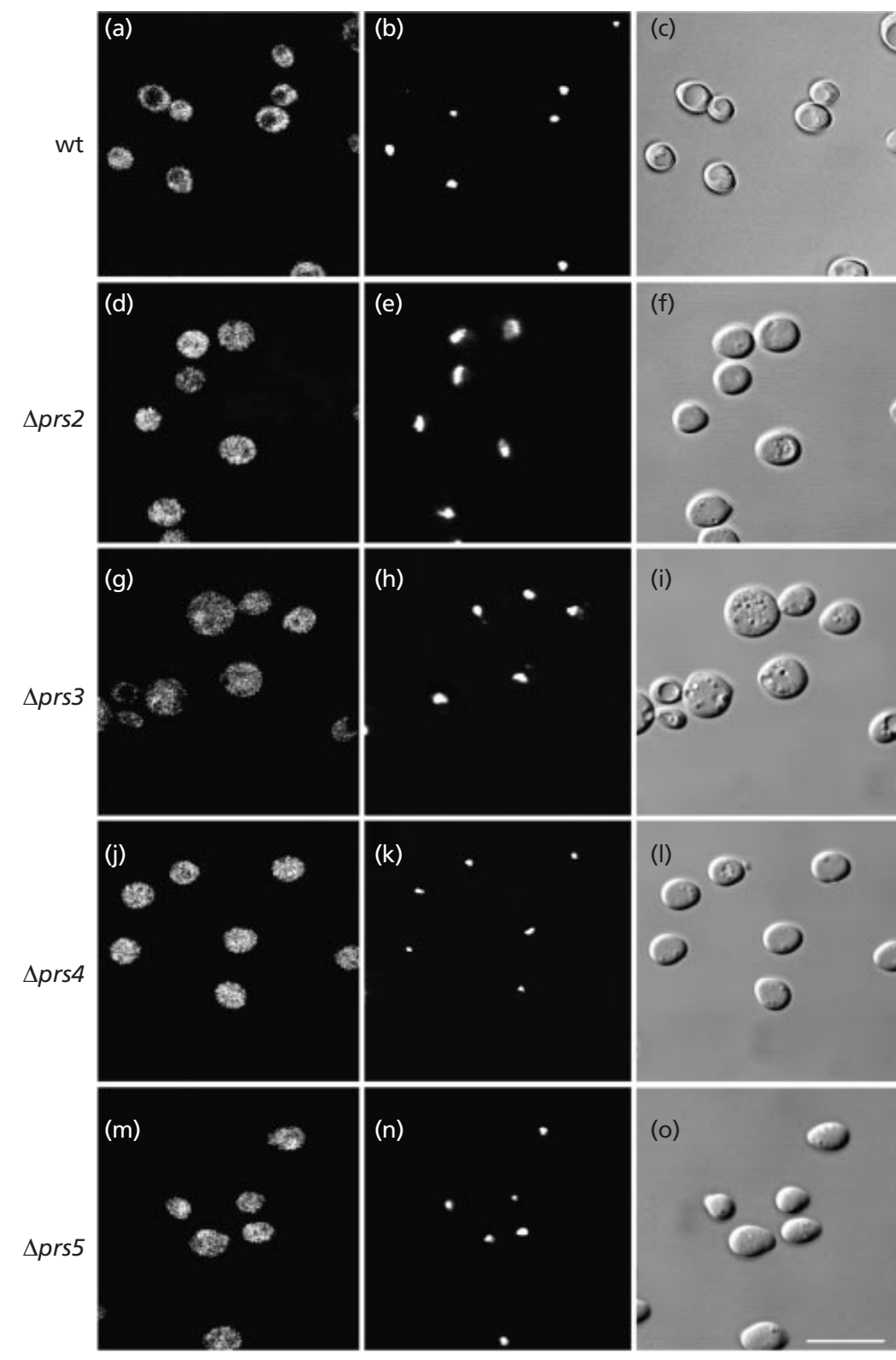

Fig. 4. Confocal analysis of the subcellular distribution of GFP-tagged Prs1p in living YN97-96 cells (wt) and strains deleted individually for PRS2 (YN98-8), PRS3 (YN9810), PRS4 (YN98-12) and PRS5 (YN98-14). The strains were grown to early exponential phase in YEPD and analysed by confocal microscopy. Confocal sections are shown in (a), (d), (g), (j) and (m) (AK39 antiserum); the corresponding DAPI images are shown in (b), (e), (h), (k) and (n); and the DIC images in (c), (f), (i), (l) and (o). Bar, $5 \mu \mathrm{m}$.
Golgi fraction (200-fold enriched for Kex2p), cytosol (enriched for GAPDH), nuclei (12-fold enriched for BiP/ Kar2p) and mitochondria (4-fold enriched for porin) were prepared by standard methods (Zinser \& Daum, 1995) and analysed for the presence of Prs1p by Western blotting. This analysis revealed that Prs1p as detected by either AK39 or AK41 was not significantly enriched in any of the fractions analysed (Fig. 3).

\section{Analysis of the subcellular localization of GFP-tagged Prs1p}

Cells expressing a GFP-Prs1p fusion protein as the only source of Prs1p grow as wild-type, indicating that the fusion protein functionally complements the lack of
Prs1p. The distribution of GFP-Prs1p in YN97-96 and in the strains containing GFP-PRS1 and a deletion of PRS2 (YN98-8), PRS3 (YN98-10), PRS4 (YN98-12) and PRS5 (YN98-14) was similar to the granular staining pattern observed with AK39 antisera in the fixed wildtype cells (Fig. 4). This result implies that deletion of PRS2, PRS3, PRS4 or PRS5 does not affect the distribution of GFP-tagged Prs1p. However, differential interference contrast (DIC) images show that all deletants had an altered morphology in comparison to the wild-type: $\Delta p r s 2$ (Fig. 4f) and $\Delta p r s 4$ (Fig. 4l) had fewer buds than the wild-type and resembled each other; $\Delta$ prs 5 strains (Fig. 4o) exhibited an elongated form and $\Delta p r s 3$ strains (Fig. 4i) appeared as highly vacuolated, large spherical cells. 

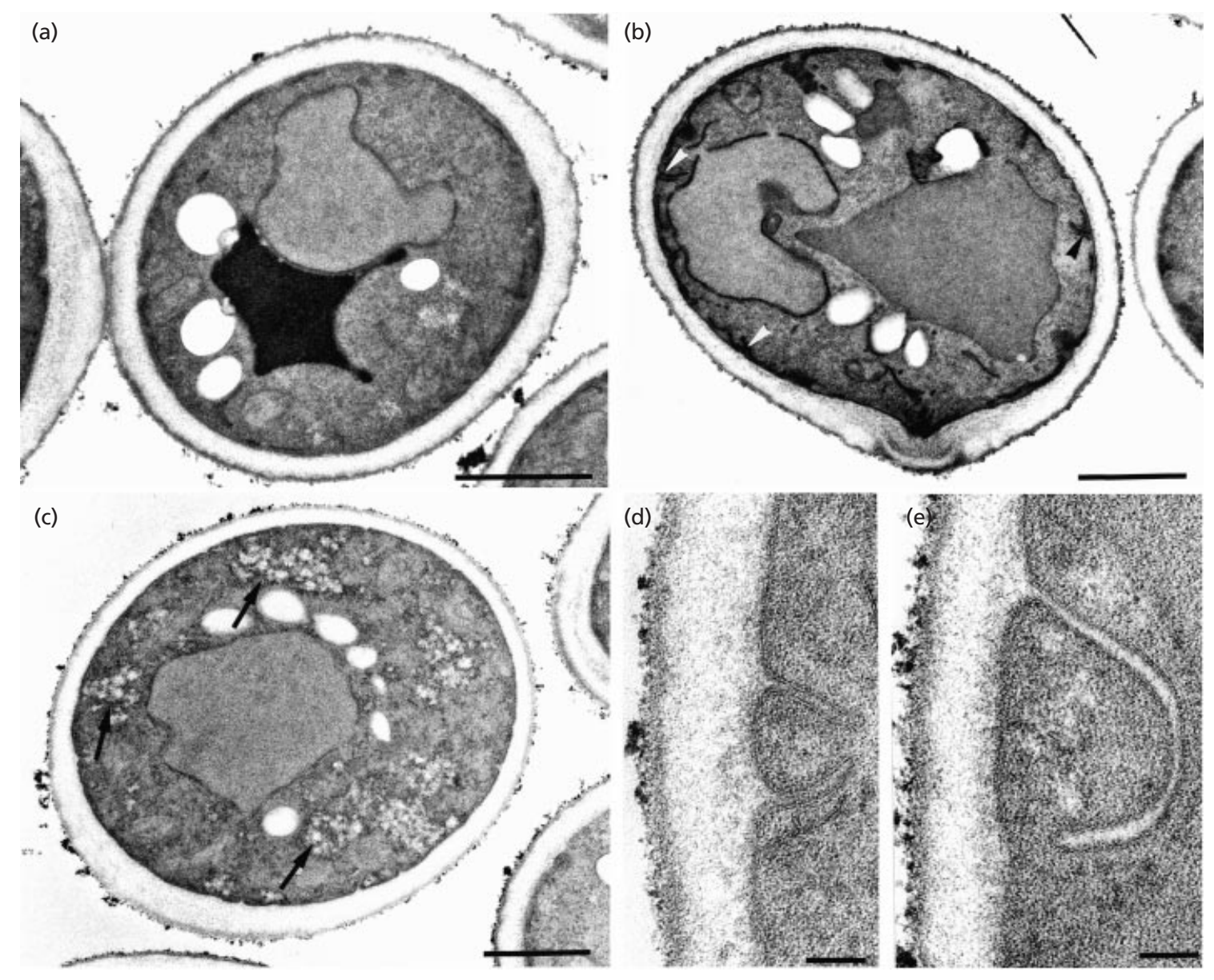

Fig. 5. Morphological analysis of wild-type and triple deletant strains. Transmission electron micrographs of wild-type (YN94-1; panel a) and triple deletant strains YN96-72 ( $\Delta p r s 2 \Delta p r s 4 \Delta p r s 5$; panel b) and YN95-25 ( $\Delta$ prs1 $\Delta$ prs3 $\Delta$ prs4; panel c). Arrowheads in (b) point to regions containing invaginations of the plasma membrane. Arrows in (c) mark cytoplasmic vesicles. Panels (d) and (e) show plasma membrane invaginations observed in YN96-72 at a higher magnification. Bars: (a-c) $1 \mu \mathrm{m}$; (d and e) $0.1 \mu \mathrm{m}$.

\section{Electron microscopic analysis of $\Delta p r s$ strains}

To gain more information about the granular cytoplasmic structures to which Prs1p localizes, we looked for ultrastructurally visible alterations in the cytoplasm that may be associated with lack of Prs1p. To this end, wild-type (YN94-1) and two triple deletants (YN96-72, $\Delta p r s 2 \quad \Delta p r s 4 \Delta p r s 5$; and YN95-25, $\Delta p r s 1 \Delta p r s 3$ aprs4) were prepared for transmission electron microscopy. The frequency of the finger-like invaginations of the plasma membrane was determined empirically and was apparently higher in the deletant strains (mean $2 \cdot 2$ per $80 \mathrm{~nm}$ thin section of a given cell; $n=35$ ) than in the wild-type (mean $1 \cdot 6 ; n=36$ ). Fig. 5 shows cross sections of the wild-type (panel a) and two triple deletant strains (panels b, c). In addition, the accumulation of cytoplasmic vesicles (panel c) appears higher in the deletant YN95-25 than in the wild-type. In panels (d) and (e), taken at a 10-fold higher magnification, side views of two invaginations of the triple deletant YN96-72 are shown.

\section{Phenotypes associated with deletion of PRS genes}

Since the only obvious phenotype of the $\Delta$ prs 1 strain was slow growth (Carter et al., 1997), we investigated some other parameters, among them sensitivity to the purine analogue caffeine. The parental strain YN94-1 was capable of growth at $6.5 \mathrm{mM}$ caffeine (Fig. 6). The growth of $\Delta p r s 1$ and $\Delta p r s 3$ was inhibited by $3.5 \mathrm{mM}$ caffeine (Fig. 6). The caffeine sensitivity of the other three single deletants is less than that of $\Delta p r s 1$ and $\Delta p r s 3$, with $\Delta p r s 5$ being inhibited by $5.0 \mathrm{mM}, \Delta p r s 4$ by $6.0 \mathrm{mM}$ and $\Delta p r s 2$ behaving like the wild-type. Certain combinations of $P R S$ deletions behaved additively, with those containing $\Delta p r s 1$ or $\Delta p r s 3$ being the most sensitive. Surprisingly, the triple deletant $\Delta p r s 1 \quad \Delta p r s 3 \quad \Delta p r s 4$ (YN95-25) was less sensitive than expected since it had the same caffeine sensitivity as the $\Delta p r s 1 \Delta p r s 4$ (YN9422) and $\Delta p r s 1 \Delta p r s 2$ (YN95-29) double deletants.

The caffeine sensitivity of the $\Delta$ prs strains could be partially alleviated when $1 \mathrm{M}$ sorbitol (Hampsey, 1997) 
Caffeine concn (mM)

$\begin{array}{lllllllllllllll}0.0 & 0.5 & 1.0 & 1.5 & 2.0 & 2.5 & 3.0 & 3.5 & 4.0 & 4.5 & 5.0 & 5.5 & 6.0 & 6.5 & 7.0\end{array}$
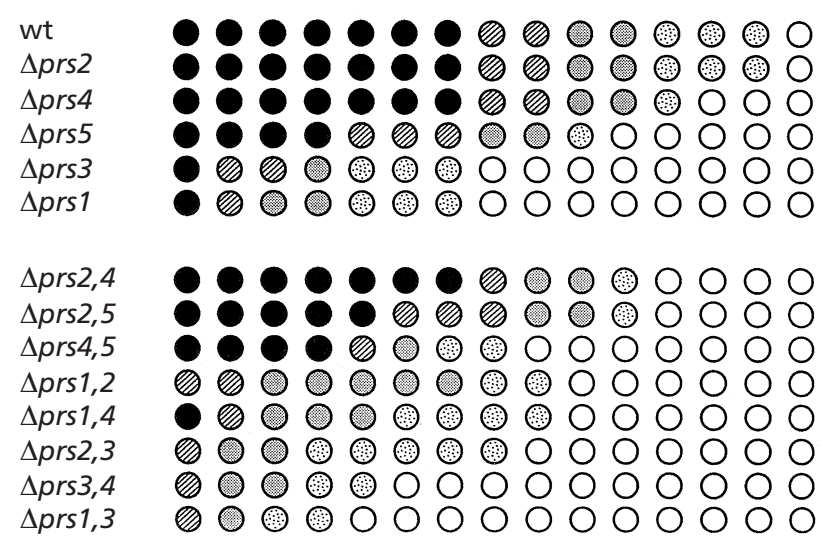

$\Delta p r s 2,4,5$

$\Delta p r s 1,3,4$

$\Delta$ prs1,2,3

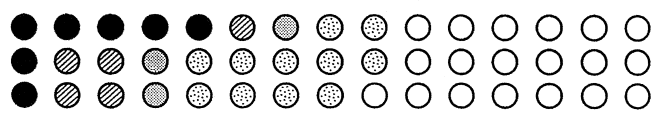

Key $\mathrm{P}^{+++} \mathrm{O}+\mathrm{O}$ papillae $\mathrm{O}-$

Fig. 6. Caffeine sensitivity of YN94-1 (wt) and $\Delta$ prs strains. The strains are labelled according to the PRS genes deleted. Caffeine concentrations in $\mathrm{mM}$ are given at the top of the figure (see Methods). The shading of the circles (see key) denotes the amount of growth obtained after $3 \mathrm{~d}$ incubation at $30{ }^{\circ} \mathrm{C}$ and the results shown represent mean scores from three independent experiments.

was included in the medium, suggesting that they might be osmotically sensitive. To test whether the $\Delta p r s$ strains were more osmotically fragile than the wild-type, the rate at which alkaline phosphatase (Posas et al., 1993) is released into the medium was measured in the presence or absence of caffeine in wild-type and several deletant strains. The rate at which alkaline phosphatase is released into the medium when the deletion strains were exposed to caffeine correlated with their sensitivity to this purine analogue (Fig. 7): in the wild-type (Fig. 7a) and in $\Delta p r s 5$ (Fig. $7 \mathrm{~b}$ ) there was only an initial release of alkaline phosphatase. However, in $\Delta$ prs 1 (Fig. 7c) and $\Delta$ prs3 (Fig. $7 \mathrm{~d}$ ) there was a sustained release of alkaline phosphatase into the medium over a period of $7 \mathrm{~h}$. This release could be prevented by performing the experiment in medium containing $1 \mathrm{M}$ sorbitol (data not shown).

\section{DISCUSSION}

Using two anti-peptide antisera, one against the NHR1-1 of Prs1p (AK39) and the other generated against another epitope of Prs1p (AK41), we determined the subcellular localization of Prs1p. Whilst it might be expected that AK41 would cross-react with other members of the PRS gene family, immunofluorescence analysis of YN94-200 [ $\Delta$ prs 1::HIS3(pYES-PRS1)] that expresses Prs1p under the control of the GAL1 promoter indicates that both antisera recognize only Prs1p, probably because the corresponding sequence differs in Prs4p in two residues, in Prs2p and Prs3p in four and in Prs5p in six residues from the 17 amino acid Prs1 peptide used to raise AK41 antisera. Furthermore, this observation is consistent with the fact that both antisera recognize a single band of approximately $54 \mathrm{kDa}$ on Western blots (Carter et al., 1997).

Immunofluorescence analysis of the subcellular distribution of Prs1p in tetraploid wild-type cells suggests that Prs1p is a cytosolic enzyme predominantly found in granular structures that localize to the cell perimeter, below the plasma membrane. This arrangement was confirmed by analysing the subcellular localization of a functional GFP-Prs1p fusion protein. The fact that Prs1p localization is not affected in secretory mutants indicates that these granular structures do not represent a post-Golgi compartment. To our knowledge, the antiPrs1p staining pattern most closely resembles that observed with an antiserum against the cell cycle protein kinase Cdc28p, which has been localized to the yeast cytoplasmic matrix (Wittenberg et al., 1987).

Western blot analysis of protein extracts of subcellular fractions isolated from yeast homogenates revealed the presence of approximately equal amounts of Prs1p in all but the mitochondrial fraction. These findings might be interpreted to suggest that Prs1p exists in an aggregated form or is otherwise associated with insoluble cytoplasmic structures. Furthermore, ultrastructural analysis of triple PRS deletion strains did not reveal any obvious alterations of the nucleo- or cytoplasm that could be associated with loss of Prs1p. However, an alteration in the cell's complement of Prs1p-Prs5p appears to have consequences for the plasma membrane as shown by the increased frequency of plasma membrane invaginations. The postulated association of Prs with the plasma membrane may also be responsible for the caffeine sensitivity observed in various deletant strains. The purine analogue caffeine affects many cellular processes; caffeine sensitivity has been shown to be associated with defects in components of the MAP (mitogen-activated protein) kinase pathways (Cid et al., 1995; Stark, 1999). $\Delta$ prs1 $\Delta p r s 3$ strains have the most caffeine-sensitive phenotype, failing to grow in the presence of $2 \mathrm{mM}$ caffeine (Fig. 6). Caffeine sensitivity can be reversed by incubation in $1 \mathrm{M}$ sorbitol as an osmotic stabilizer (Hampsey, 1997). Interestingly, PRS3 was isolated in a colony-sectoring assay to identify genes interacting with WHI2 whose deletion also causes caffeine sensitivity. However, Binley et al. (1999) showed that mutants of PRS3 and WHI2 are not co-lethal. The same authors have documented that strains lacking $\Delta$ prs 3 are affected not only in their ability to grow in the presence of $5 \mathrm{mM}$ caffeine but also do not respond normally to nutrient deprivation, and are altered in ion homeostasis and in the organization of the actin cytoskeleton in stationary phase. The varying degrees of caffeine sensitivity observed in the PRS deletant strains may reflect inability to maintain cell integrity as suggested by the microscopic analysis of the plasma 

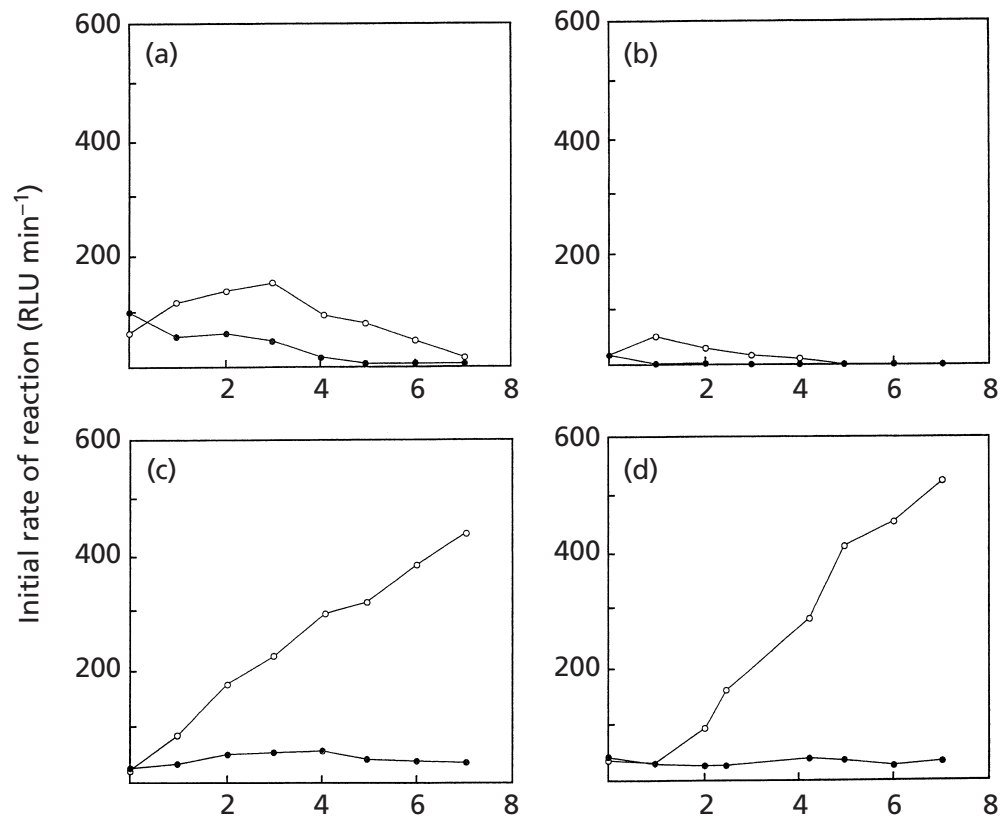

Time (h)

Fig. 7. Deletion of $P R S$ genes affects release of alkaline phosphatase. Strains were grown to $\mathrm{OD}_{600} 1.0$ in YEPD medium and the cultures divided into two equal portions. Caffeine at a final concentration of $5 \mathrm{mM}$ was added to one aliquot $(O)$ and an equivalent amount of sterile distilled $\mathrm{H}_{2} \mathrm{O}$ was added to the other (O). The cells were incubated further and samples removed hourly over a period of $7 \mathrm{~h}$. The strains used were (a) wild-type (YN94-1), (b) YN96-1

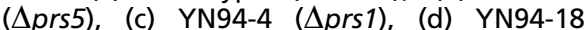
$(\Delta p r s 3)$. Results shown are initial rates of reaction $\left(\mathrm{RLU} \mathrm{min}^{-1}\right)$. membrane (Fig. 5). Further evidence of impairment of cell integrity is afforded by the demonstration that the loss of either PRS1 or PRS3 leads to the release of alkaline phosphatase in the presence of caffeine (Fig. 7). This caffeine-associated release of alkaline phosphatase is prevented by the addition of $1 \mathrm{M}$ sorbitol (data not shown). Deletants $\Delta p r s 2, \Delta p r s 4$ and $\Delta p r s 5$, which are only slightly more sensitive to caffeine than the wildtype, do not show a caffeine-associated release of alkaline phosphatase. The osmotic remediability of the caffeine-sensitive phenotypes of certain PRS deletant strains and the release of alkaline phosphatase suggest that in some way an alteration of the Prs complement of the cell can affect cell integrity.

The results presented here and elsewhere (Hernando et al., 1999) suggest that the products of the five PRS genes could aggregate to form subcellular structures which may play a role in maintaining cell integrity. If one assumes that single and multiply deleted strains contain Prs complexes different from the wild-type, this could explain the alteration in caffeine sensitivity as reflecting a suboptimal maintenance of cell integrity.

\section{ACKNOWLEDGEMENTS}

The work was supported by grants from the BBSRC (UK) and EC EUROFAN programme to M.S., and from the Austrian Lise-Meitner Foundation and the Swiss National Science Foundation Project 823A-046702 to R.S. We thank G. Daum, A. Ivessa and B. M. Pearson for helpful discussions throughout the course of this work; G. Daum for access to his collection of antisera and strains; A. Ivessa, R. Leber, H. Pichier and A. Pump for samples of subcellular fractions; and R. Schekman for anti-invertase antiserum. We also thank J. C. Slaughter for critical reading of the manuscript.

\section{REFERENCES}

Becker, M. A., Puig, J. G., Mateos, F. A., Jimenez, M., Kim, M. \& Simonds, H. A. (1988). Inherited superactivity of phosphoribosylpyrophosphate synthetase: association of uric acid overproduction and sensorineural deafness. Am J Med 85, 383-390.

Becker, M. A., Smith, P. R., Taylor, W., Mustafi, R. \& Switzer, R. L. (1995). The genetic and functional basis of purine nucleotide feedback-resistant phosphoribosylpyrophosphate synthetase superactivity. J Clin Invest 96, 2133-2141.

Binley, K. M., Radcliffe, P. A., Trevethick, J., Duffy, K. A. \& Sudbery, P. E. (1999). The yeast PRS3 gene is required for cell integrity, cell cycle arrest upon nutrient deprivation, ion homeostasis and the proper organization of the actin cytoskeleton. Yeast 15, 1459-1469.

Boeke, J., Trueheart, J., Natsoulis, G. \& Fink, G. R. (1987). 5Fluoro-orotic acid as a selective agent in yeast molecular genetics. Methods Enzymol 154, 164-175.

Carter, A. T., Narbad, A., Pearson, B. M., Beck, K.-F., Logghe, M., Contreras, R. \& Schweizer, M. (1994). Phosphoribosylpyrophosphate synthetase $(P R S)$ : a new gene family in Saccharomyces cerevisiae. Yeast 10, 1031-1044.

Carter, A. T., Beiche, F., Hove-Jensen, B., Narbad, A., Barker, P. J., Schweizer, L. M. \& Schweizer, M. (1997). PRS1 is a key member of the gene family encoding phosphoribosylpyrophosphate synthetase in Saccharomyces cerevisiae. Mol Gen Genet 254, 148-156.

Cid, V. J., Durán, A., del Rey, F., Snyder, M. P., Nombela, C. \& Sánchez, M. (1995). Molecular basis of cell integrity and morphogenesis in Saccharomyces cerevisiae. Microbiol Rev 59, 345-386.

Daum, G., Böhni, P. C. \& Schatz, G. (1982). Import of proteins into mitochondria. Cytochrome $b_{2}$ and cytrochrome $c$ peroxidase are located in the intermembrane space of yeast mitochondria. J Biol Chem 257, 13028-13033.

Gaigg, G., Simbeni, R., Hrastnik, C., Paltauf, F. \& Daum, G. (1995). Characterization of a microsomal subfraction associated with 
mitochondria of the yeast, Saccharomyces cerevisiae. Biochim Biophys Acta 1234, 214-220.

Gietz, R. D. \& Woods, R. A. (1994). High efficiency transformation with lithium acetate. In Molecular Genetics of Yeast. A Practical Approach, pp. 121-131. Edited by J. R. Johnson. Oxford: IRL Press.

Güldener, U., Heck, S., Fiedler, T., Beinhauer, J. \& Hegemann, J. H. (1996). A new efficient gene disruption cassette for repeated use in budding yeast. Nucleic Acids Res 24, 2519-2524.

Hampsey, M. (1997). A review of phenotypes in Saccharomyces cerevisiae. Yeast 13, 1099-1133.

Heim, R. \& Tsien, R. Y. (1996). Engineering green fluorescent protein for improved brightness, longer wavelengths and fluorescence resonance energy transfer. Curr Biol 6, 178-182.

Hernando, Y., Parr, A. \& Schweizer, M. (1998). PRS5, the fifth member of the phosphoribosyl pyrophosphate synthetase gene family in Saccharomyces cerevisiae, is essential for viability in the absence of either PRS1 or PRS3. J Bacteriol 180, 6404-6407.

Hernando, Y., Carter, A. T., Parr, A., Hove-Jensen, B. \& Schweizer, M. (1999). Genetic analysis and enzyme activity suggest the existence of more than one minimal functional unit capable of synthesising phosphoribosyl pyrophosphate in Saccharomyces cerevisiae. J Biol Chem 274, 12480-12487.

Hove-Jensen, B. (1989). Phosphoribosylpyrophosphate (PRPP)less mutants of Escherichia coli. Mol Microbiol 3, 1487-1492.

Hurt, E. C., McDowall, A. \& Schimmang, T. (1988). Nucleolar and nuclear envelope proteins of the yeast Saccharomyces cerevisiae. Eur J Cell Biol 46, 554-563.

Kadowaki, T., Schneiter, R., Hitomi, M. \& Tartakoff, A. M. (1995). Mutations in nucleolar proteins lead to nucleolar accumulation of poly $(\mathrm{A})^{+}$RNA in Saccharomyces cerevisiae. Mol Cell Biol 6, 1103-1110.

Kaiser, C., Michaelis, S. \& Mitchell, A. (1994). Methods in Yeast Genetics. Cold Spring Harbor, NY: Cold Spring Harbor Laboratory.

Khorana, H. G., Fernandes, J. F. \& Kornberg, A. (1958). Pyrophosphorylation of ribose-5-phosphate in the enzymatic synthesis of 5phosphorylribose 1-pyrophosphate. J Biol Chem 230, 941-948.

Leber, A., Hrastnik, C. \& Daum, G. (1995). Phospholipidsynthesizing enzymes in Golgi membranes of the yeast Saccharomyces cerevisiae. FEBS Lett 377, 271-274.

Novick, P., Field, C. \& Schekman, R. (1980). Identification of 23 complementation groups required for post-translational events in the yeast secretory pathway. Cell 21, 205-215.

Pearson, B. M., Hernando, Y. \& Schweizer, M. (1998). Construction of PCR-ligated long flanking homology cassettes for use in the functional analysis of six unknown open reading frames from the left and right arms of Saccharomyces cerevisiae chromosome XV. Yeast 14, 391-399.

Posas, F., Casamayor, A. \& Ariño, J. (1993). The PPZ protein phosphatases are involved in the maintenance of osmotic stability of yeast cells. FEBS Lett 318, 282-286.

Rapoport, T. A., Jungnickel, B. \& Kutay, U. (1996). Protein transport across the eucaryotic endoplasmic reticulum and bacterial inner membranes. Annu Rev Biochem 65, 271-303.
Sakakibara, Y. (1992). dnaR function of the prs gene of Escherichia coli in initiation of chromosome replication. J Mol Biol 226, 989-996.

Sambrook, J., Fritsch, E. F. \& Maniatis, T. (1989). Molecular Cloning: a Laboratory Manual, 2nd edn. Cold Spring Harbor, NY : Cold Spring Harbor Laboratory.

Schekman, R. (1985). Protein localization and membrane traffic in yeast. Annu Rev Cell Biol 1, 115-143.

Serrano, R. (1988). $\mathrm{H}^{+}$-ATPase from plasma membranes of Saccharomyces cerevisiae and Avena sativa roots: purification and reconstitution. Methods Enzymol 157, 533-544.

Sikorski, R. S. \& Hieter, P. (1989). A system of shuttle vectors and yeast host strains designed for efficient manipulation in Saccharomyces cerevisiae. Genetics 122, 19-27.

Stark, M. J. R. (1999). Protein phoshorylation and dephosphorylation. In The Metabolism and Molecular Physiology of Saccharomyces cerevisiae, pp. 209-275. Edited by J. R. Dickinson \& M. Schweizer. London: Taylor \& Francis.

Switzer, R. L. (1969). Regulation and mechanism of phosphoribosylpyrophosphate synthetase. I. Purification and properties of the enzyme from Salmonella typhimurium. J Biol Chem 244, 2854-2863.

Switzer, R. L. \& Sogin, D. C. (1973). Regulation and mechanism of phosphoribosylpyrophosphate synthetase. V. Inhibition by end products and regulation by adenine diphosphate. J Biol Chem 248, 1063-1073.

Taira, M., lizasa, T., Shimida, H., Kudoh, J., Shimizu, N. \& Tatibana, M. (1990). A human testis-specific mRNA for phosphoribosyl-pyrophosphate synthetase that initiates from a nonAUG codon. J Biol Chem 265, 16491-16497.

Tatibana, M. (1995). Mammalian phosphoribosyl-pyrophosphate synthetase. Adv Enzyme Regul 35, 229-249.

Wach, A., Brachat, A., PohImann, R. \& Philippsen, P. (1994). New heterologous modules for classical or PCR-based gene disruptions in Saccharomyces cerevisiae. Yeast 10, 1793-1808.

Wach, A., Brachat, A., Alberti-Segui, C., Rebischung, C. \& Philippsen, P. (1997). Heterologous HIS3 marker and GFP reporter modules for PCR-targeting in Saccharomyces cerevisiae. Yeast 13, 1065-1075.

Wente, S. R., Rout, M. P. \& Blobel, G. (1992). A new family of yeast nuclear pore complex proteins. J Cell Biol 119, 705-723.

Whitters, E. A., McGee, T. P. \& Bankaitis, V. A. (1994). Purification and characterization of a late Golgi compartment from Saccharomyces cerevisiae. J Biol Chem 269, 28106-28117.

Wittenberg, C., Richardson, S. L. \& Reed, S. I. (1987). Subcellular localization of a protein kinase required for cell cycle initiation in Saccharomyces cerevisiae: evidence for an association between the CDC28 gene product and the insoluble cytoplasmic matrix. J Cell Biol 105, 1527-1538.

Zinser, E. \& Daum, G. (1995). Isolation and biochemical characterization of organelles from the yeast Saccharomyces cerevisiae. Yeast 11, 493-536.

Received 10 April 2000; revised 8 August 2000; accepted 21 August 2000. 